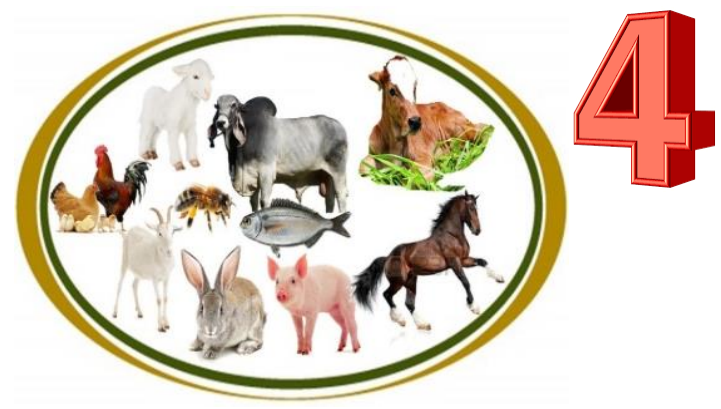

Recepción: 16 de abril de 2020

\section{Ofacultad de Giencias Agropecuarias}

ISSN L 2644-3856

Revista Investigaciones Agropecuarias

Volumen 2, $N^{\circ}$ 2. pp. 49-68

Junio 2020 - Noviembre 2020

Panamá

Aceptación: 4 de mayo de 2020

\title{
PERFIL DE LA CURVA DE LACTACIÓN Y PRODUCCIÓN LÁCTEA COMERCIAL EN VACAS CEBUIINAS Y CRUZADAS EN FINCAS LECHERAS CON BAJA TECNOLOGÍA EN EL TRÓPICO
}

\author{
Edil E. Araúz', Eduardo E. Araúż y Ángela Montezuma
}

1* Universidad de Panamá, Facultad de Ciencias Agropecuarias, Departamento de Zootecnia. Panamá

${ }^{2}$ Ingeniero Agronomo Zootecnista, Servicios profesionales y asesorías en sistemas e industria de la Producción Animal, Bioseguridad, Procesamiento y Conservacion de los Productos de Origen Animal. Panamá

${ }^{3}$ Zootecnista y Extensionista en Ganadería, Ministerio de Desarrollo Agropecuario, Región 1 en la Provincia de Chiriquí, Panamá. @ *edil.arauz@up.ac.pa, eduarauz 20@hotmail.com, angelamontezuma25@gmail.com

\section{RESUMEN}

La curva de lactación y la producción de leche comercial fueron evaluadas en 250 vacas multíparas cebuínas y cebuínas x europeo tipo leche en catorce fincas lecheras Grado C; bajo pastoreo, estrés calórico moderado y un ordeño manual diario con ternero en la época lluviosa en el trópico húmedo de bajura; donde el estrés calórico diurno máximo ocurrió a la 01 PM (HR $76.71 \%$, TAbs $32.7^{\circ} \mathrm{C}$, RSD $676.3 \mathrm{kcal} / \mathrm{m}^{2} \mathrm{hr}$, ITH 86.59 e ITH RSDVVmax 92.35). La producción comercial acumulada y la longitud lactacional en el grupo cebuíno resultó en $692.07 \mathrm{~kg}$ en 177 días; $1 / 2$ Bos taurus tipo leche x $1 / 2$ Cebú $1267.35 \mathrm{~kg}$ en 213 días y en las $3 / 4$ Bos taurus tipo leche $\mathrm{x} 1 / 4$ Cebú $2012.16 \mathrm{~kg}$ en 262 días $(\mathrm{P}<.01)$. El perfil típico de la curva de lactación mostró una producción diaria inicial, máxima y final en las cebuínas de 3.42, 4.62 a los 14 días y $2.23 \mathrm{~kg} / \mathrm{vaca}(\mathrm{P}<.05) ; 5.63,8.63$ a los 26 días y $3.27 \mathrm{~kg} / \mathrm{vaca}(\mathrm{P}<.01)$ en las $1 / 2$ Bos taurus tipo leche $\mathrm{x} 1 / 2$ Cebú y $7.50,10.95$ a los 34 días y $4.40 \mathrm{~kg} / \mathrm{vaca}(\mathrm{P}<.01$ en las 3/4Bos taurus x 1/4 Cebú). La curva de lactación en las cebuínas fue $\mathrm{Y}_{(\mathrm{kg} / \mathrm{vaca}-\text {-día })}=13.09572121 \mathrm{X}^{-}$ ${ }_{0.0733187} \mathrm{e}^{-0.005064242 \mathrm{X}}\left(\mathrm{R}^{2}=0.38\right)$; en las $1 / 2$ Cebú $\mathrm{x}{ }^{1 / 2}$ Bos taurus $\mathrm{Y}_{(\mathrm{kg} / \text { vaca-día })}=8.5935 \mathrm{X}^{\mathbf{0 . 1 3 0 4 8}} \mathrm{e}^{-}$ ${ }_{0.005115067 X}\left(R^{2}=0.68\right)$ y en las $3 / 4$ Bos taurus lechero x $1 / 4$ Cebú $Y_{(\mathrm{kg} / \text { vaca-día })}=12.7632 \mathrm{X}^{\mathbf{0 . 2 4 0 5 1}} \mathrm{e}^{-}$ $0.007117078 \mathrm{X}\left(\mathrm{R}^{2}=0.72\right)$. La producción lechera fue potenciada con el mayor aporte genético tipo leche en los cruces; sin embargo, el entorno ambiental y el manejo afectaron la curva de lactación y redujeron la habilidad lechera fenotípica en todos los grupos genéticos en el trópico húmedo.

PALABRAS CLAVES: cebuínas, cruzadas, estrés calórico, lactación, lecherías, trópico, potencial lechero 


\section{PROFILE OF THE LACTATION CURVE AND COMERTIAL MILK PRODUCTION IN ZEBU AND CROSSBRED COWS IN LOW TECHNOLOGYCAL SMALL DAIRY FARMS IN THE TROPIC}

\section{ABSTRACT}

The lactation curve and commertial milk production were studied in 250 lactating multiparous zebu and crossbred cows (zebu x Bos taurus milk type) in 14 small dairy farms Grade C under grayzing, mild heat stress, calf stimulation and one milking by day across raining time in lowland of humid tropic. The maximum heat stress occurred at $01 \mathrm{PM}$ (RH 76.71\%, $\mathrm{AT}_{\mathrm{db}}$ $32.7^{\circ} \mathrm{C}$, DSR $676.3 \mathrm{kcal} / \mathrm{m}^{2} \mathrm{hr}$, THI 86.59 and THIDSRWS 92.35). Total commertial milk production and lactation length resulted for zebu $692.07 \mathrm{~kg}$ in 177 days, $1 / 2$ Bos taurus milk type $\mathrm{x} 1 / 2$ Zebu 1267.35 in 213 days and for $3 / 4$ Bos taurus type milk x $1 / 4$ Zebu $2012.16 \mathrm{~kg}$ in 262 days $(\mathrm{P}<.01)$. The tipic profile of the lactation curve in the zebu group showed initial, peak and final daily milk yield of 3.42, 4.62 at 14 days and $2.23 \mathrm{~kg} / \mathrm{cow}(\mathrm{P}<.05)$; in $1 / 2$ Bos taurus milk type $\mathrm{x} 1 / 2$ Zebu 5.63, 8.63 at 26 days $(\mathrm{P}<.01)$ and in 3/4Bos taurus milk type $\mathrm{x} 1 / 4$ Zebu 7.50, 10.95 at 34 days and $4.40 \mathrm{~kg} / \mathrm{cow}(\mathrm{P}<.01)$. The lactation curve resulted in Zebu cows $\mathrm{Y}_{(\mathrm{kg} / \text { cow-day })}=13.09572121 \mathrm{X}^{-\mathbf{0 . 0 7 3 3 1 8 7}} \mathrm{e}^{-\mathbf{0 . 0 0 5 0 6 4 2 4 2 X}}\left(\mathrm{R}^{2}=0.38\right)$; in $1 / 2 \mathrm{Zebu} \mathrm{X} 1 / 2$ Bos taurus $\mathrm{Y}_{(\mathrm{kg} / \mathrm{cow}-\mathrm{day})}=8.5935 \mathrm{X}^{0.13048} \mathrm{e}^{-0.005115067 \mathrm{X}}\left(\mathrm{R}^{2}=0.68\right)$ and in $3 / 4$ Bos taurus lechero $\mathrm{x} 1 / 4$ Zebu $\mathrm{Y}_{(\mathrm{kg} / \mathrm{cow}-\mathrm{day})}=12.7632 \mathrm{X}^{\mathbf{0 . 2 4 0 5 1}} \mathbf{e}^{-0.007117078 \mathrm{X}}\left(\mathrm{R}^{2}=0.72\right)$. Milk production was potentiated by the proportion of Bos taurus milk type in the crossbred; however, the environment and management altered the lactation curve and reduced phenotype milk ability in all genetic groups in the humid tropical climate.

KEYWORDS: crossbred, dairy farms, heat stress, lactation, tropic, milk potential, Zebu 


\section{INTRODUCCIÓN}

La producción lechera tropical presenta varios modelos tecnológicos de fincas; entre ellas, que poseen las menores condiciones en infraestructuras, facilidades físicas, inversiones, tipo de alimentación basada en el forraje verde a través del pastoreo, sistema de registros simplificados o ausentes, animales con bajo potencial genético lechero, ausencia de equipos para la preservación y el cuidado de la leche (Araúz y Guevara, 2015). Estas fincas han sido clasificadas de acuerdo con la legislación sanitaria y de calidad láctea en Panamá en Grado C; siendo el mayor grupo de productores que alcanzaron unas 5197 (89.73\% de las fincas lecheras en Panamá entre el 2010 y 2011) según el MIDA (2012); las cuales representan en la actualidad unas 6453 fincas (MIDA, 2018).

Las fincas lecheras Grado C producen el 47\% de la producción nacional en Panamá; al mismo tiempo que contribuyen como fuente de trabajo y sustento económico para las familias rurales y constituyen un apoyo para el funcionamiento y la operación de las pequeñas y medianas industrias de lácteos nacionales (MIDA, 2012, 2018). Sin embargo, con la evolución de la situación lechera en Panamá, las fincas con la menor tecnología han mostrado fortalezas y ventajas operativas ya que tienen menores inversiones fijas, animales más resistentes al trópico, menor dependencia en la mano de obra, menores costos de producción y un menor compromiso financiero (Araúz, 2019). A pesar de la prevalencia de las fincas lecheras Grado C en Panamá y otros países latinoamericanos; aún no se cuenta con estudios sectoriales nacionales que describan el potencial lechero y la curva de lactación; destacando aspectos como: producción inicial, máxima y al cierre, tiempo al pico lactacional, persistencia y tiempo en producción. El objetivo de esta investigación fue establecer cuáles son los descriptores críticos de la curva de lactación en vacas multíparas cebuínas y cruzadas cebú x europeo tipo leche (Holstein, Pardo Suizo); así como su rendimiento lechero potencial con fines comerciales con baja tecnología lechera, alimentación a base de forraje verde, un ordeño manual diario con ternero y periodo de acceso al amamantamiento libre de unas cinco horas.

\section{Características e indicadores de la Curva de lactación y el potencial lechero en la vaca}

El ciclo de vida biológico de la hembra bovina incluye los sectores del crecimiento, desarrollo, pubertad, establecimiento reproductivo efectivo, la gestación, el parto, la lactación, la nueva

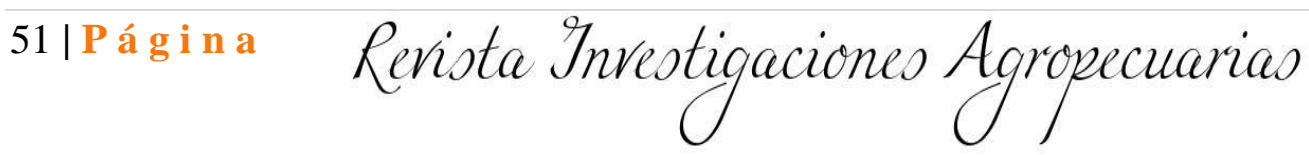




\section{Ofracultad de Giencias Agropecuarias}

gestación, el periodo del secado y el nuevo parto y que luego de iniciar la fase reproductiva se describe como el intervalo entre partos (Araúz, 2019). Este ciclo es importante para las acciones y directrices del manejo apropiado en nutrición, reproducción, salud, genética, ambiente, bienestar y bioseguridad (Crowe et al., 2018).

La trayectoria de la curva de lactación es esencial para establecer el manejo optimo en el manejo nutricional, reproductivo y sanitario de la vaca lechera moderna para hacer los modelos de producción más eficientes fisiológicamente y alcanzar una mayor rentabilidad económica (Cabrera, 2018).

Uno de los modelos más utilizados para describir la curva de lactación en la vaca ha sido la función gamma incompleta descrita por la expresión: $\mathrm{Y}_{\mathrm{t}}=\mathrm{a} \mathrm{t}{ }^{\mathrm{b}} \mathrm{e}^{-\mathrm{ct}}$ donde $\mathrm{Yt}$ es la producción de leche diaria en kilogramos, a es la producción al inicio de la lactación, t es el tiempo lactación en días, b es la pendiente hacia la máxima producción y c es la pendiente del máximo al cierre lactacional y e es el valor neperiano 2.718282 como modelo integral (Wood, 1967). El modelo linearizado ln $(y t)=\ln (a)+b \ln (t)-c t$ permite estimar el tiempo para la máxima producción láctea $(b / c)$, la máxima producción $\left(\mathrm{a}(\mathrm{b} / \mathrm{c}) \mathrm{b}_{\mathrm{e}-\mathrm{b})}\right.$ y la persistencia láctea $[-(\mathrm{b}+1) \ln (\mathrm{c})]$ según Atashi et al., (2012). La producción de leche es afectada por varios factores, los cuales modifican la curva lactacional; sobresaliendo el plano nutricional (Walker et al., 2004) y el estrés calórico que afecta la producción (Gupta et al., 2016; Das et al., 2016) y la reproducción (West, 2003; Hansen, 2009; Crowe y Williams, 2018) debido a las alteraciones en la fisiología general, el metabolismo, la carga calórica corporal y en la cinética de la termorregulación (Araúz, 2017).

Los modelos regresivos lactacionales predicen la trayectoria de la producción de leche y contribuyen con la adecuación del manejo y el aprovechamiento de la capacidad productiva en las fincas lecheras (Cabrera, 2018). Por ende, los registros biolactacionales son esenciales para evaluar la capacidad funcional de las vacas y definir el manejo integral; en especial en el medio trópico, donde hay limitantes nutricionales y el estrés térmico que reducen simultáneamente la productividad lechera (Araúz, 2010).

\section{Requerimientos energéticos y alimentación de la vaca lechera en el trópico}

El patrón de los requerimientos nutricionales energéticos para el ganado lechero ha sido establecido por la NRC (2001) y la ARC (1999); a partir de las cuales se han derivado las

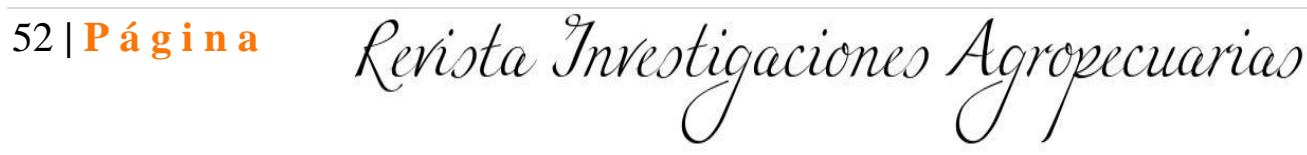


normativas de alimentación y los ajustes del manejo para potenciar la capacidad lechera de los bovinos según su valor genético. El perfil energético para la producción de leche aun para la ganadería con baja tecnología lechera es un factor de valor productivo (Araúz, 2015).

Existe un balance favorable para la producción lechera tropical a través de las lecherías grado C ya que la alimentación depende del forraje verde, los animales presentan un menor tamaño corporal (McDowell, 1981), la habilidad para utilizar la energía para producir leche es baja (NRC, 2001) y el perfil genético favorece el almacenamiento de reservas corporales y limita la movilización de los nutrientes dietéticos y endógenos para la producción de leche (Stothard et al., 2004). La habilidad del tejido mamario, el patrón endocrino y el perfil metabólico se encuentra bajo el control de los genes que regulan el metabolismo del tejido adiposo y el uso de otras reservas durante la lactación en las hembras cebuínas; limitando el uso de nutrientes para la biosíntesis láctea (Nayeri y Stothard, 2016).

El gasto energético para la locomoción en el ganado lechero es $0.00045 \mathrm{Mcal} / \mathrm{kg}$ por cada kilómetro recorrido y para el pastoreo 0.0012 Mcal por cada kg de peso corporal (NRC, 2001). En el trópico se gasta energía extra para realizar la termólisis obligatoria que demanda la homeotermia para evitar el distanciamiento calórico extremo corporal y la alteración normotérmica (McNab, 2001). Las condiciones del trópico incluyen el estrés calórico efectivo durante la fase diurna (Araúz, 2010); lo que altera el balance calórico y el bienestar biotérmico (Polsky et al., 2017). En consecuencia, el pastoreo, la locomoción y la termólisis de la vaca en producción demandan energía que reduce la habilidad lechera (Araúz, 2006).

El periodo de lactación representa la fase metabólica en la que se utilizan las reservas orgánicas (grasa corporal, músculo y huesos); así como las reservas hepáticas (glucógeno, vitaminas liposolubles) y minerales óseos que son utilizadas para la biogénesis de la leche; dependiendo del potencial lechero, del estado lactacional y de la composición de la leche (Knegsel et al., 2007). Las vacas cebuínas y cruzadas Holstein x cebuínas bajo diferentes perfiles de alimentación y nutrición energética restringida mostraron que utilizan la energía a medida que aumenta la producción lechera (Araujo et al., 2018).

Los forrajes tropicales son reconocidos por su bajo valor energético para favorecer la producción lechera al contener entre 0.12 y $0.35 \mathrm{Mcal} / \mathrm{kg}$ de EN leche; incluyendo los pastos de corte y las gramíneas pasto señal (Brachiaria decumbens) y el pasto estrella (Cynnodon dactylon) según 53 | Pá g i in a

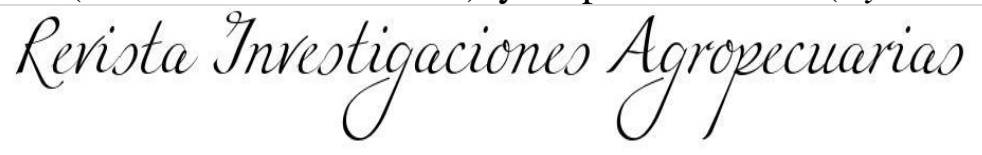


varios estudios (Rosas et al., 1985). La presión energética sobre las reservas corporales durante la lactación en el trópico está acompañada de un rápido deterioro de la condición corporal de la vaca en producción aun en las fincas lecheras grado C (Araúz, 2018).

\section{PARTE EXPERIMENTAL}

\section{Ubicación del estudio y área geográfica}

El estudio fue desarrollado en el Distrito de Alanje (El Tejar, Santo Thomas, Canta Gallo, La Pita, Mostrenco, Querébalos, La Martina y Guarumal) en la Provincia de Chiriquí; utilizando catorce fincas lecheras Grado C en la época lluviosa del 2008 al 2012.

\section{Animales experimentales para la medición lactacional y los registros de producción}

Se utilizó 250 vacas multíparas entre dos y cuatro partos de un total de 355 animales candidatos que fueron distribuidas en cebuínas 58, 1/2 Cebú x 1/2 Europeo tipo leche 106 y 3/4 Europeo tipo leche x $1 / 4$ Cebú 86. El tipo lechero incluyó los aportes de la raza Holstein y Pardo Suizo como parte de los cruzamientos. Se estableció el registro de la producción de leche semanal con la identificación individual según la fecha postpartal de producción.

\section{Peso corporal, peso metabólico, superficie corporal, alimentación y manejo en el ordeño}

El peso corporal fue determinado por perimetría torácica utilizando la cinta OVNY de tela y plástico resistente confeccionada en Colombia (Inalmet, 2007) calibrada para cebuínos y bovinos cruzados de doble propósito.

El peso metabólico determinó según la NRC (2001), la superficie corporal para cebuínos según Curtis (1981) para los cebuínos $\mathrm{SC}_{\left(\mathrm{M}^{2}\right)}=0.12\left(\mathrm{PC}_{\mathrm{kg}}\right)^{0.60}$; mientras que para los $1 / 2$ Cebuínos $\mathrm{x} 1 / 2$ Europeo Lechero $\left(\mathrm{SC}_{\left(\mathrm{M}^{2}\right)}=0.135\left(\mathrm{PC}_{\mathrm{kg}}\right)^{0.58}\right)$ y en los 3/4 Bos taurus lechero $\mathrm{x} 1 / 4$ Cebuíno $\mathrm{SC}_{\left(\mathrm{M}^{2}\right)=}$ 0.143(PC kg) $)^{0.57}$ según Araúz (2018).

El manejo animal incluyó acceso al pastoreo libre en el día y en la noche; con acceso a las crías entre las 8 AM y 01 PM); separando los terneros a la 01 PM hasta el siguiente día en la mañana. El manejo incluyó el amamantamiento restringido, higiene de los pezones con agua clorinada, el ordeño manual y el pesaje de la producción cada siete días. La alimentación dependió del forraje verde con pastoreo libre y se utilizó el pasto suazi (Digitaria swazilandensis), señal (Brachiaria decumbens) y en algunas fincas (3) se utilizó la asociación suazi y pangola (Digitaria 
decumbens Stent). Se utilizó sal mineralizada (Sal Cruda 65\%) y una fuente de Minerales en un $35 \%$ ) a base de calcio, fósforo, magnesio, manganeso, zinc, hierro, cobre, cobalto y zinc complementada con vitaminas (Laboratorios Bayer, 2015).

\section{Entorno microambiental, sensibilidad y presión calórica ambiental}

El medio microambiental de las fincas fue evaluado en el campo abierto; tomando una medición psicrométrica en la mañana (06 am) y en la tarde a la 01:00 PM cada 15 días en la época lluviosa. Se utilizó el termohigrómetro digital para registrar la Temperatura Ambiental de bulbo seco $\left({ }^{\circ} \mathrm{C}\right)$ y la Humedad Relativa (\%) en cada finca codificada para conformar la base de datos microclimáticos. La evaluación psicrométrica (Martínez, 2012), el ITH según Armstrong, (1994) y Dikmen y Hansen (2006) y el ITHRSDVV según Mader et al. (2006). La radiación solar directa y la velocidad del viento se obtuvieron en Unión Fenosa para Alanje. Los índices de sensibilidad calórica normal y ajustada se determinaron según: $\mathrm{ITH}=($ TAbs x 0.80 $)+[($ HR/100 x $($ TAbs 14.4)] e ITHRSDVV $=4.51+$ ITH $-\left(1.992 \times \mathrm{VV}_{\mathrm{m} \mathrm{seg}-1}\right)+\left(0.0068 \mathrm{x} \mathrm{W}^{-2} \mathrm{hr}^{-1}\right)$.

\section{Medición de la Producción de Leche y el contenido de grasa láctea}

Se peso la producción de leche obtenida por ordeño manual diario, registrada según la finca, la vaca y la fecha a través del formulario de campo; generando 8168 observaciones para la producción de leche (1508 en cebuínos, 3392 en 1⁄2 Bos taurus tipo leche x 1/2 Cebú y 3268 en 3/4 Bos taurus tipo leche $\mathrm{x} 1 / 4$ Cebú). Se registró el peso del recipiente vacío, el recipiente más la leche obtenida y el peso de la leche $(\mathrm{kg})$; utilizando una romana digital marca Constant (HQ), seriada 14192-115E con capacidad de $50 \mathrm{~kg}$. La fecha de parto de cada animal y la producción de leche fue anotada a partir del $8^{\mathrm{vo}}$ al $10^{\mathrm{mo}}$ día postparto hasta culminar su ordeño comercial. Una muestra de leche fue tomada cada 14 días de cada vaca para el análisis de la grasa láctea, analizada en el laboratorio de Fisiología Animal y Ciencia Lechera del Departamento de Zootecnia.

\section{Diseño Experimental y Análisis Estadístico}

El modelo utilizado incluyo: años $\left(A_{i}\right)$, grupos genéticos $\left(B_{k}\right)$, estado lactacional semanal $\left(C_{m}\right)$ y las covariables pesos corporal en la primera semana de producción y el cambio de temperatura ambiental diurna; conformando el arreglo factorial más covarianza; descrito por como:

$\mathbf{Y}_{\mathrm{ijk}}=\mathbf{u}+\mathbf{A}_{\mathbf{i}}+\mathbf{B}_{\mathrm{k}}+\mathbf{C}_{\mathrm{m}}+(\mathbf{B C})_{\mathrm{ik}}+\mathbf{b}_{1}\left(\mathbf{X}_{\mathrm{ij}}-\hat{\mathbf{u}}_{1}\right)+\mathbf{b}_{2}\left(\mathbf{X}_{\mathrm{ij}}-\hat{\mathbf{u}}_{2}\right)+\mathbf{e}_{\mathrm{ikj}} \quad$ donde

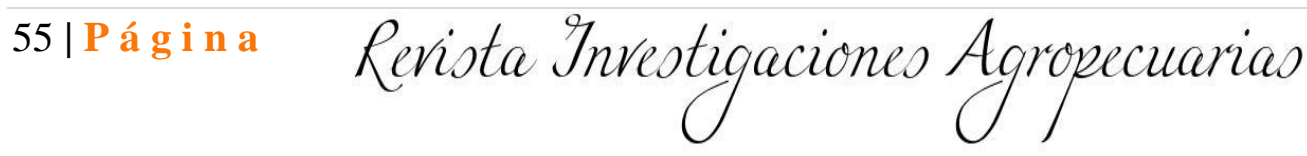


$\mathrm{Y}_{\mathrm{ijk}}=$ es la producción de leche diaria del animal $\mathrm{j}^{\mathrm{mo}}$ en el año $\mathrm{i}^{\mathrm{mo}}$, en el grupo genético $\mathrm{k}^{\mathrm{mo}}$ en función del estado lactacional $\mathrm{m}^{\mathrm{mo}}$ intervenido por el peso corporal $(\mathrm{kg})$ en la primera semana de producción y el cambio térmico diurno $\left({ }^{\circ} \mathrm{C}\right)$ entre las $7 \mathrm{AM}$ y la $1 \mathrm{PM}$.

$\mathrm{u}=$ Media general

Ai: Efecto del $\mathrm{i}^{\mathrm{mo}}$ año del estudio ( $\mathrm{i}^{\mathrm{mo}}=1$ (2008), 2(2009), 3(2010), 4(2011), 5(2012).

Bk: grupo genético $\left(\mathrm{k}^{\mathrm{mo}}=\right.$ cebuínas (1), 1/2Cebu x 1/2 taurina (2) y 3/4BTTL x 1/4Cebu (3)

$\mathrm{Cm}$ : Estado lactacional semanal $\left(\mathrm{m}^{\mathrm{mo}}=1, \ldots . ., 37^{\circ}\right.$ semanas de producción láctea diaria)

$(\mathrm{CB})_{\mathrm{km}}$ : Interacción del Grupo genético x el Estado lactacional semanal

$\mathrm{b}_{1}\left(\mathrm{Xij}-\hat{\mathrm{u}}_{1}\right)$ : Peso corporal de la vaca en la primera semana de producción comercial en $\mathrm{kg}$.

$\mathrm{b}_{2}\left(\mathrm{Xij}-\hat{u}_{2}\right)$ : Cambio térmico diurno en ${ }^{\circ} \mathrm{C}$ cada 15 días.

$\mathrm{e}_{\mathrm{ijkm}}=$ Residuo o Error Experimental; incluyendo el efecto de fincas en el residuo o error.

Las medias cuadradas fueron ajustadas por covarianza y se utilizó el error tipo III para ajustar el análisis de varianza. La curva de lactación por grupos genético fue generada según la Función Logística Gamma Incompleta (Wood, 967): $\mathrm{Y}_{\mathrm{t}(\mathrm{kg} / \mathrm{día})}=\mathrm{at}^{\mathrm{b}} \mathrm{e}^{-\mathrm{c}}$; siendo $\mathrm{Y}$ la producción de leche diaria $(\mathrm{kg})$ en el momento lactacional $\mathrm{t}$ (día), a es la producción de leche inicial (comercial), b es la pendiente desde el inicio al máximo de producción, c es la pendiente del pico al cierre lactacional y e es el número neperiano; integrados según la programación en SAS (2004) descrita por Herrera y Barreras (2001).

\section{RESULTADOS Y DISCUSIÓN}

\section{Microambiente, presión calórica y estrés térmico efectivo}

El entorno microambiente diurno en la época lluviosa para el periodo en estudio mostró una temperatura ambiental promedio antes de la salida del sol de $23.5^{\circ} \mathrm{C}$, Humedad Relativa $88.3 \%$, ITH 73.33 e ITH ajustado 73.74; indicando la menor presión calórica y la menor influencia térmica para los bovinos (Tabla I). Para la 01 Pm se alcanzó un comprometimiento térmico microambiental 56|Pág in a Revista Investigaciones Agropecuarias 
(TAbs $32.7^{\circ} \mathrm{C}$, HR 76.7\%, ITH 86.59 e ITH ajustado 92.35) que represento un estrés calórico severo; aunque es evidente que la tensión calorica trasciende a partir de las 10 en adelante; siendo perjudicial para la salud térmica y productiva de la vaca lechera según Dikmen y Hansen (2009). La modificación térmico ambiental (AM vs PM) fue de $+25.23 \%$; incluyendo la contribución de la temperatura ambiental, la humedad relativa y la radiación solar directa (Tabla 1). La mayor presión y estrés calórico se alcanzó después del medio día, cuyo nivel fue severo con las implicaciones negativas en la fisiología sistémica, la termorregulación, la conducta y la producción lechera (West, 2003; Araúz et al., 2015; Polsky et al., 2017). Por ende, se requieren ajustes en el manejo y medio físico en las horas más calurosas para cuidar el bienestar térmico endógeno, reducir los efectos sistémicos negativos (Araúz, 2006; Arias et al., 2008), evitar o reducir las alteraciones metabólicas (Slimen et al., 2016; Gupta et al., 2016), reproductivas (Jordan et al., 2003; Hansen, 2009; Das et al., 2016) y las pérdidas económicas implícitas (St-pierre et al., 2003).

Tabla 1. Indicadores del perfil microclimático diurno promedio en el Distrito de Alanje en la época lluviosa durante el estudio lactacional.

Puntos críticos del Horario microclimático

\begin{tabular}{|c|c|c|c|c|}
\hline Indicador Microclimático & Unidad & $\begin{array}{l}\text { 6:00 AM } \\
\text { (Mínimo) }\end{array}$ & $\begin{array}{l}\text { 01:00 PM } \\
\text { (Máximo) }\end{array}$ & $\begin{array}{c}\Delta \\
\text { Microclimático } \\
\text { Térmico }\end{array}$ \\
\hline Temperatura del aire Bulbo seco & ${ }^{\circ} \mathrm{C}$ & 23.5 & 32.7 & +9.2 \\
\hline Temperatura del aire Bulbo Húmedo* & ${ }^{\circ} \mathrm{C}$ & 22.05 & 29.10 & 7.05 \\
\hline Punto de Roció* & ${ }^{\circ} \mathrm{C}$ & 21.46 & 28.06 & +6.60 \\
\hline Humedad Relativa & $\%$ & 88.3 & 76.7 & -11.6 \\
\hline Entalpia* & $\mathrm{KJ} / \mathrm{kg}$ as & 64.01 & 94.90 & +30.89 \\
\hline Volumen Específico* & $\mathrm{M}^{3} / \mathrm{kg}$ as & 0.862 & 0.90 & +0.038 \\
\hline Presión Atmosférica* & Kpa & 1013.25 & 1013.25 & ------- \\
\hline Radio de Humedad* & $\mathrm{g} \mathrm{H}_{2} \mathrm{O} / \mathrm{kg}$ as & 0.0161 & 0.0242 & +0.0081 \\
\hline Presión Parcial de Vapor de Agua* & $\mathrm{Pa}$ & 2558 & 3798 & +1.24 \\
\hline Presión de vapor de agua Saturada* & $\mathrm{Pa}$ & 2897 & 4950 & 2053 \\
\hline Densidad Específica del aire * & $\mathrm{M}^{3} / \mathrm{kg}$ & 1.18 & 1.14 & -.04 \\
\hline Radiación Solar Directa ${ }^{\ddagger}$ & $\mathrm{Kcal} / \mathrm{m}^{2} \mathrm{hr}$ & 0.000 & 676.3 & +676.3 \\
\hline Velocidad del Viento $\neq$ & $\mathrm{Km} / \mathrm{hr}$ & 7.41 & 7.41 & ---- \\
\hline $\begin{array}{l}\text { Indice Temperatura }- \text { Humedad } \\
\text { (ITH) }{ }^{\dagger}\end{array}$ & ${ }^{\circ} \mathrm{C} \%$ & 73.33 & 86.59 & $+13 . \overline{2} 6$ \\
\hline ITHRSDVV $\left({ }^{\circ} \mathrm{C} \% \mathrm{Wm}^{-2} \mathrm{Hr}^{-1} \mathrm{~m}^{-1} \mathrm{seg}^{-1}\right) \uparrow$ & & 73.74 & 92.35 & +18.61 \\
\hline
\end{tabular}

Araúz, E. E. (2010, 2018) ‡NOAA (2014) *Knigth, J. (2006, 2020) † (Mader et al., 2006) 


\section{Peso corporal y metabólico y estado hematimétrico de las vacas experimentales}

La categorización del peso corporal al parto en las vacas multíparas fue diferente entre los tres grupos genéticos $(\mathrm{P}<.01)$; evidenciando que a medida que aumentó la contribución genética del Bos taurus tipo leche en los cruces también aumentó el peso corporal, el peso metabólico y la superficie corporal (tabla II). El perfil hematimétrico no fue diferente para el hematocrito entre los grupos genéticos ( $\mathrm{P}>.05)$; sin embargo, el hematocrito, hemoglobina y el conteo de glóbulos rojos mostraron una ligera disminución al aumentar el aporte genético del ganado taurino lechero en los cruzamientos; siendo la hemoglobina y el conteo de glóbulos rojos menores ante los cebuínos ( $\mathrm{P}<.05)$. McDowell (1985) indica que las vacas cebuínas pesan $350 \mathrm{~kg}$ a diferencia del presente estudio con $402 \mathrm{~kg}$; siendo superior en un 14.85\%. Las vacas media sangre promediaron $437 \mathrm{~kg} \mathrm{y}$ el grupo con más contribución lechera pesó $478 \mathrm{~kg}$ (Tabla 2); siendo inferior en 16.8\%. Estas diferencias obedecen a la influencia del ambiente; formando parte de la variación fenotípica que desarrollan los bovinos cebuínos y cruzados en las condiciones tropicales; donde el forraje verde es la principal fuente de alimentación y el estrés calórico se combinan; moldeando la biológico de la vaca cebuíno y cruzada bajo el perfil subnutricional y la adaptación somática para minimizar la influencia negativa del estrés térmico.

Tabla 2. Indicadores somáticos y hematimétricos al parto según el grupo genético.

\begin{tabular}{|c|c|c|c|}
\hline \multirow[b]{2}{*}{ Composición del Grupo Genético } & \multicolumn{3}{|c|}{ Grupo Genético de las Vacas multíparas en lactación } \\
\hline & $\begin{array}{c}\text { Cebuínas } \\
\mathbf{y} \\
\text { Acebuadas }\end{array}$ & $\begin{array}{c}1 / 2 \text { Bos taurus } \\
\mathbf{X} \\
1 / 2 \text { Cebú }\end{array}$ & $\begin{array}{c}3 / 4 \text { Bos taurus } \\
\mathbf{X} \\
{ }_{1 / 4} \text { Cebú }\end{array}$ \\
\hline Peso Corporal al Parto (kg) & $402 \pm 37^{a}$ & $437 \pm 46^{\mathrm{b}}$ & $478 \pm 51^{\mathrm{c}}$ \\
\hline Peso Metabólico al parto $\left(\mathrm{kg}^{3 / 4}\right)$ & $89.77 \pm 15.00$ & $95.58 \pm 17.66$ & $102.22 \pm 19.08$ \\
\hline Superficie Corporal $\left(\mathrm{m}^{2}\right)$ & $4.38 \pm 1.05 \mathrm{a}$ & $4.59 \pm 1.24^{\mathrm{b}}$ & $4.82 \pm 1.34^{\mathrm{c}}$ \\
\hline Hematocrito Venoso (\%) & $31.75 \pm 3.13^{\mathrm{a}}$ & $30.65 \pm 2.75^{\mathrm{a}}$ & $29.78 \pm 2.70^{\mathrm{a}}$ \\
\hline Hemoglobina (g/100 ml) & $11.16 \pm 1.06^{\mathrm{a}}$ & $11.03 \pm 1.15^{\mathrm{a}}$ & $10.45 \pm 1.48^{\mathrm{b}}$ \\
\hline Conteo de Eritrocitos $\left(10^{6} / \mathrm{ml}\right)$ & $6.36 \pm 0.51^{\mathrm{a}}$ & $6.04 \pm 0.97^{b}$ & $5.87 \pm 0.98^{c}$ \\
\hline
\end{tabular}

Medias a b y b c difieren al 5\% (P<.05); a c difieren al 1\% (P<.01); letras iguales no difieren al 5\% (P>.05)

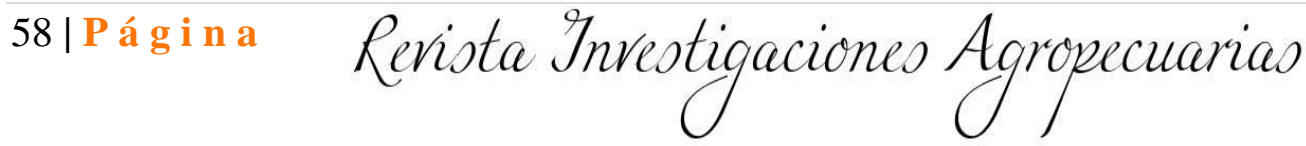




\section{Producción de leche comercializada y prevalencia biolactacional en las fincas}

La producción de leche comercializada fue heterogénea a través de fincas evaluadas debido a las contribuciones de la disponibilidad del forraje verde, tipo de pastura, planificación forrajera y manejo de las pasturas, composición genética de los animales y composición genética de las vacas en producción al momento de la evolución. El potencial lechero de cada finca lechera Grado C fue considerado en un ordenamiento secuencial en base al promedio diario potencia por vaca; pudiendo observarse que un total de cuatro fincas (28.35\%) mostraron una producción diaria de 3.12 a $3.97 \mathrm{~kg} / \mathrm{vaca}$; constituyendo las fincas de menor potencial lechero. Las fincas intermedias oscilaron entre 4.04 y $4.94 \mathrm{~kg} / \mathrm{vaca}$ e incluyeron un total de seis fincas que representaron el 42.86\%. El tercer grupo de fincas lecheras grado C presentó un rango diario promedio entre 5.4 y $9.81 \mathrm{~kg} / \mathrm{vaca}$ y representaron el $28.57 \%$ de las unidades ganaderas evaluadas. Las fincas estuvieron en el mismo entorno microclimático, pero la variación de la producción lechera diaria comercial fue $39.18 \%$ con un promedio de $5.23 \pm 2.05 \mathrm{~kg} / \mathrm{vaca}$ (ver Figura 1 ).

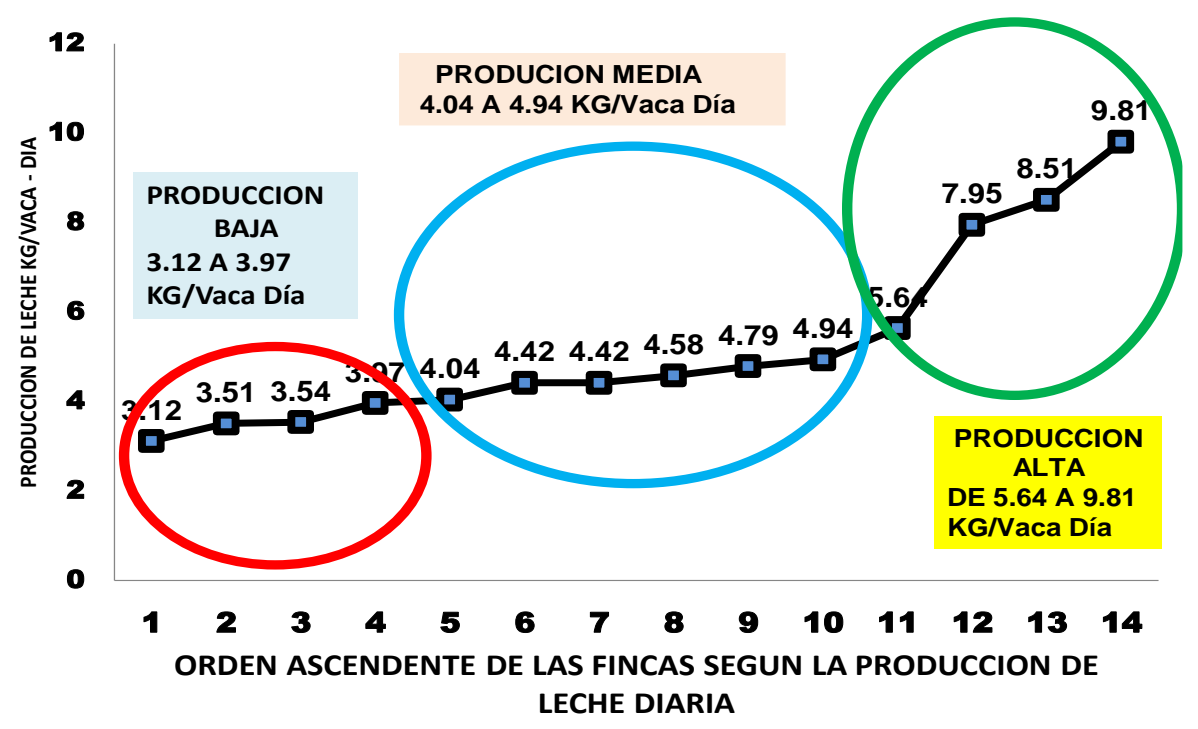

Figura 1. Ubicación de las fincas lecheras Grado C evaluadas en el distrito de Alanje.

La ponderación e importancia de la producción lechera diaria en las fincas Grado C representa un aporte esencial en los sistemas ganaderos tropicales con tecnología limitada ya que los ingresos dependen de la comercialización de la leche y de la venta de los terneros luego del destete (Guerra, 59|Pági a Revista Investigaciones Agropecuarias 


\section{Ofracultad de Giencias Agropecuarias}

2015). Sin embargo, estos resultados indican que los ingresos y la productividad ganadera se encuentra afectada por la baja habilidad lechera de orden genético; además de confrontar las limitaciones nutricionales de los pastos tropicales (Rosas et al.,1988, 1994), los efectos directos del estrés calórico en la fase diurna (Araúz, 2006, 2017), la influencia negativa de una baja frecuencia del ordeño (Gómez, 2002) y la práctica de permitir el a amamantamiento por cuatro a cinco horas en las vacas en producción con el propósito de alimentar parcialmente las crías (Araúz, 2019). Estos factores se conjugan alrededor de la ganadería lechera de doble propósito y conducen a la generación de la baja productividad ganadera en Panamá (Corella, 2016).

\section{Indicadores de la curva de lactación y la producción de leche total comercializada}

El empleo de la función gamma incompleta sobre la producción de leche y el estado lactacional permitió determinar los descriptores de la curva de lactación; los cuales fueron diferentes entre los grupos genéticos $(\mathrm{P}<.001)$; así como la duración lactacional y la producción láctea comercial ( $\mathrm{P}<.001)$. La producción de leche inicial, máxima y al cierre en las cebuínas fue 3.42, 5.58 y 2.33 $\mathrm{kg} /$ día; en las vacas $1 / 2$ Cebú x 1/2 Bos taurus tipo leche fueron $5.63,8.63$ y $3.27 \mathrm{~kg} /$ día; y en el grupo 3/4 Bos taurus tipo leche x 1/4 Cebú resultó en 7.5, 10.95 y $4.4 \mathrm{~kg} /$ día; evidenciando la mayor diferencia al aumentar el potencial lechero (ver Tabla 3 ).

Las inflexiones diferenciales al inicio de la lactación de los cruces de media y tres cuartos ganado europeo tipo leche $\mathrm{x}$ cebuínos superaron la producción inicial cebuína en 64.5 y 128\%; sin embargo, al considerar los máximos de producción absoluta, se observó un incremento de 54.66 en el cruce $1 / 2$ Bos taurus tipo leche x $1 / 2$ Cebú y de $96.24 \%$ en las vacas producto del cruce $3 / 4$ Bos taurus tipo leche $\mathrm{x} 1 / 4$ Cebú. El cierre lactacional en las cebuínas ( $2.33 \mathrm{~kg} / \mathrm{vaca}$ día) fue superado por las vacas $1 / 2$ Bos taurus tipo leche $\mathrm{x} 1 / 2$ Cebú en $40 \%$ y en las $3 / 4$ Bos taurus tipo leche $\mathrm{x} 1 / 4$ Cebú en un $89 \%$ sobre las cebuínas como referentes (Figura 2).

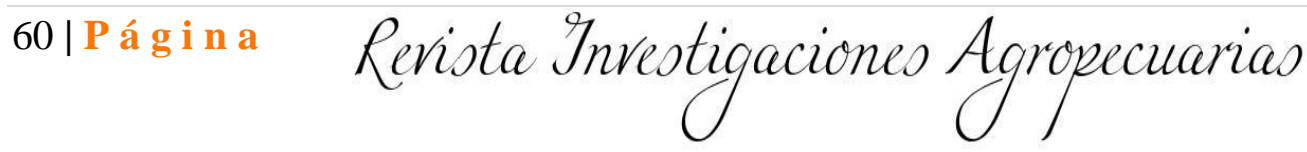




\section{(5)racultad de Giencias Agropecuarias}

Tabla 3. Descriptores lactacionales según el grupo genético en lecherías Grado C.

\begin{tabular}{lccc} 
& \multicolumn{3}{c}{ Grupo Genético de las Vacas en lactación } \\
\hline Indicadores Lactacionales & Cebuínas & $\begin{array}{c}1 / 2 \text { Bos taurus } \mathbf{x} \\
1 / 2 \text { Cebú }\end{array}$ & $\begin{array}{c}3 / 4 \text { Bos taurus } \mathbf{x} \\
1 / 4 \text { Cebú }\end{array}$ \\
\hline Producción inicial (kg) & $3.42 \pm 0.49^{\mathrm{a}}$ & $5.63 \pm 0.88^{\mathrm{b}}$ & $7.50 \pm 0.85^{\mathrm{c}}$ \\
Producción Máxima (kg) & $5.58 \pm 0.61^{\mathrm{a}}$ & $8.63 \pm 0.84^{\mathrm{b}}$ & $10.95 \pm 1.17^{\mathrm{c}}$ \\
Producción al Cierre (kg) & $2.33 \pm 0.53^{\mathrm{a}}$ & $3.27 \pm 0.65^{\mathrm{b}}$ & $4.40 \pm 0.70^{\mathrm{c}}$ \\
Tiempo a la máxima producción (Días) & $14^{\mathrm{a}}$ & $26^{\mathrm{b}}$ & $34^{\mathrm{c}}$ \\
Duración lactacional (Días) & $177^{\mathrm{a}}$ & $217^{\mathrm{b}}$ & $262^{\mathrm{c}}$ \\
Producción de Leche Comercial (kg) & $692.07^{\mathrm{a}}$ & $1267.35^{\mathrm{b}}$ & $2012.16^{\mathrm{c}}$ \\
Producción Láctea comercial al 4\% (kg) & $798.99^{\mathrm{a}}$ & $1,339.59^{\mathrm{b}}$ & $1933.69^{\mathrm{c}}$ \\
Grasa Láctea lactacional (\%) & $5.03 \pm 1.12^{\mathrm{a}}$ & $4.38 \pm 0.89^{\mathrm{b}}$ & $3.74 \pm 0.58^{\mathrm{c}}$ \\
\hline
\end{tabular}

Medias con las letras a b y b c entre los grupos genéticos difieren al $1 \%(\mathrm{P}<.01)$.

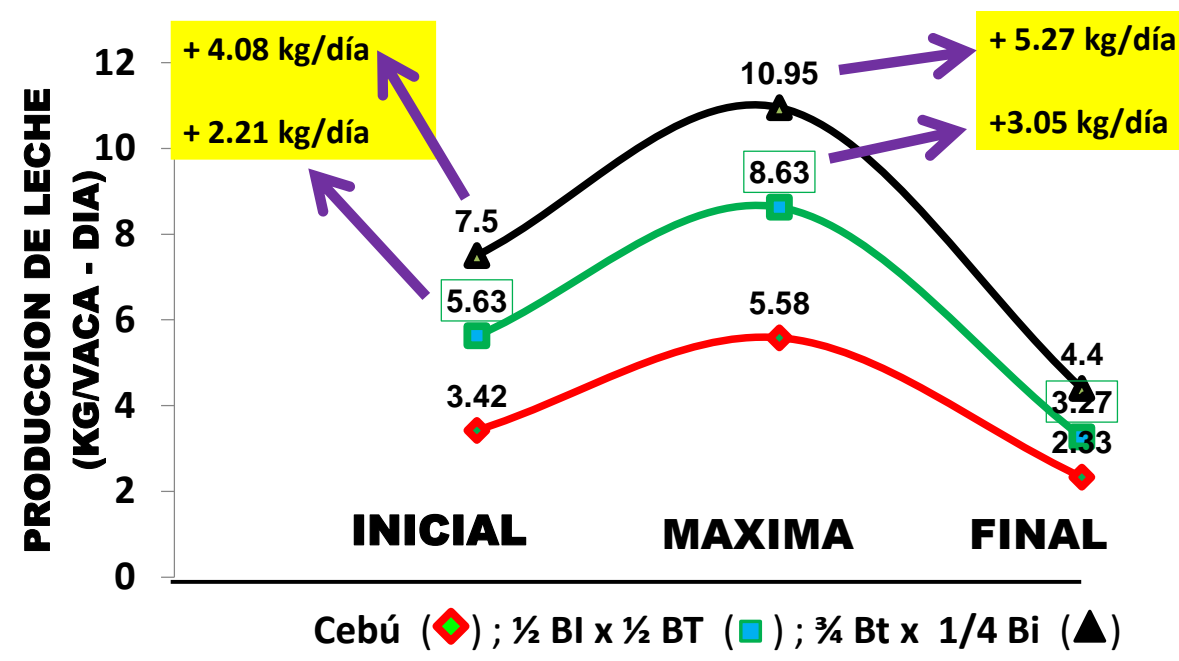

Figura 2. Valor crítico referente en la producción lechera inicial, máxima y al final de la lactación en fincas lecheras Grado C según el grupo genético y fenotípico.

La curva de lactación fue diferente entre los grupos genéticos; destacando que el periodo de producción fue más corto ocurrió en las cebuínas (177 \pm 12 días); mientras que en las vacas $1 / 2$ Bos taurus tipo leche $\mathrm{x} 1 / 2$ Cebú fue $213 \pm 16.75$ días; siendo ambos superados por las vacas $3 / 4$ Europeo x 1/4 Cebú con $262 \pm 21.14$ días (Tabla 4). El término comercial se refiere al periodo que las vacas $61 \mid$ Pági a Revista Investigaciones Agropecuarias

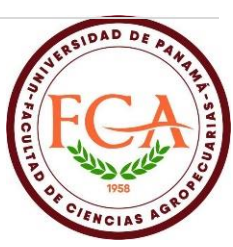


se mantuvieron en producción para la venta de leche; aunque las mismas continuaron con sus crías como parte del perfil de manejo en las lecherías grado $\mathrm{C}$.

La producción de leche y la longitud lactacional fueron diferentes entre los grupos genéticos $(\mathrm{P}<.001 ; \mathrm{P}<.04)$ y según el estado lactacional $(\mathrm{P}<.001)$. La producción láctea total de índole comercial por vaca resultó en las cebuínas $692.07 \mathrm{~kg}$ en 177 días, 1/2Cebú x 1/2 Bos taurus lechero $1267.35 \mathrm{~kg}$ en 213 días y en las 3/4 Bos taurus tipo leche x 1/4 cebú $2012.16 \mathrm{~kg}$ en 262 días con un ordeño manual diario y acceso al amamantamiento por la cría $(\mathrm{P}<.001)$.

La producción de leche comercial aumentó entre las vacas cebuínas y las 1/2 Cebú x 1/2 Bos taurus tipo leche en $+575.28 \mathrm{~kg}(+1.8312 \mathrm{X})$ con un diferencial lactacional de +40 días $(+1.2259 \mathrm{X})$; mientras que el grupo genético 3/4 Bos taurus tipo leche x 1/4 Cebú aumentó con respecto a las cebuínas en $+1320.00 \mathrm{~kg}(+2.908 X)$ en 262 días de producción. La grasa láctea disminuyó desde el grupo cebuíno hasta el grupo con la mayor influencia genética taurina tipo leche. La producción láctea ajustada al 4\% de grasa resultó en $798.99 \mathrm{~kg}$ en las cebuínas; en las $1 \frac{1}{2}$ Bos taurus lechero x 1/2 Cebú $1339.59 \mathrm{~kg}$ y en las 3/4 Bos taurus tipo leche x 1/4 Cebú fue $1933.69 \mathrm{~kg}$. La producción de leche acumulada para las vacas $1 \frac{2}{2}$ Bos taurus tipo leche x 1/2 Cebú según McDowell (1985) fue $2830 \mathrm{~kg}$ contra $1267.35 \mathrm{~kg}$ en 213 días (44.78\%); mientras que el grupo con más genética lechera alcanzó 2012.16 kg (39.53\%) de 5090 kg.

Tabla 4. Componentes de curva de lactación según la función gamma incompleta para las vacas cebuínas, $1 / 2$ cebú x 1/2 Bos taurus y $1 / 2$ Bos taurus lechero $x$ 1/2 Cebuínas.

\begin{tabular}{lccc} 
& \multicolumn{3}{c}{ Grupos Genéticos } \\
\cline { 2 - 4 } Indicadores Lactacionales & Cebuínas & $\begin{array}{c}1 / 2 \text { Bos taurus x } \\
\mathbf{1} / \mathbf{2} \text { Cebú }\end{array}$ & $\begin{array}{c}\mathbf{3} \mathbf{4} \text { Bos taurus } \mathbf{x} \\
\mathbf{1} \mathbf{4} \text { Cebú }\end{array}$ \\
\hline Componente a & 13.09572121 & 8.59350000 & 12.76320000 \\
Componente b & -0.073327187 & 0.13048000 & 0.240510000 \\
Componente c & -0.005064242 & -0.005115067 & -.007117078 \\
Tiempo a la máxima producción (días) & 14 & 26 & 34 \\
Producción de leche máxima (lb/día) & 10.14 & 11.51 & 17.29 \\
Persistencia Láctea (lbs) & 4.822 & 6.087 & 6.350 \\
Longitud Lactacional (días) & $177 \pm 12.12$ & $213 \pm 16.75$ & $262 \pm 21.14$ \\
Coeficiente R ${ }^{2}$ ajustado & 0.38 & 0.68 & 0.72 \\
Observaciones Lactacionales Semanales & 1508 & 3392 & 3268 \\
\hline
\end{tabular}

62|Págin a Revista Investigaciones Agropecuarias

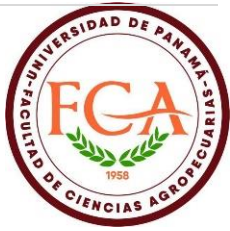




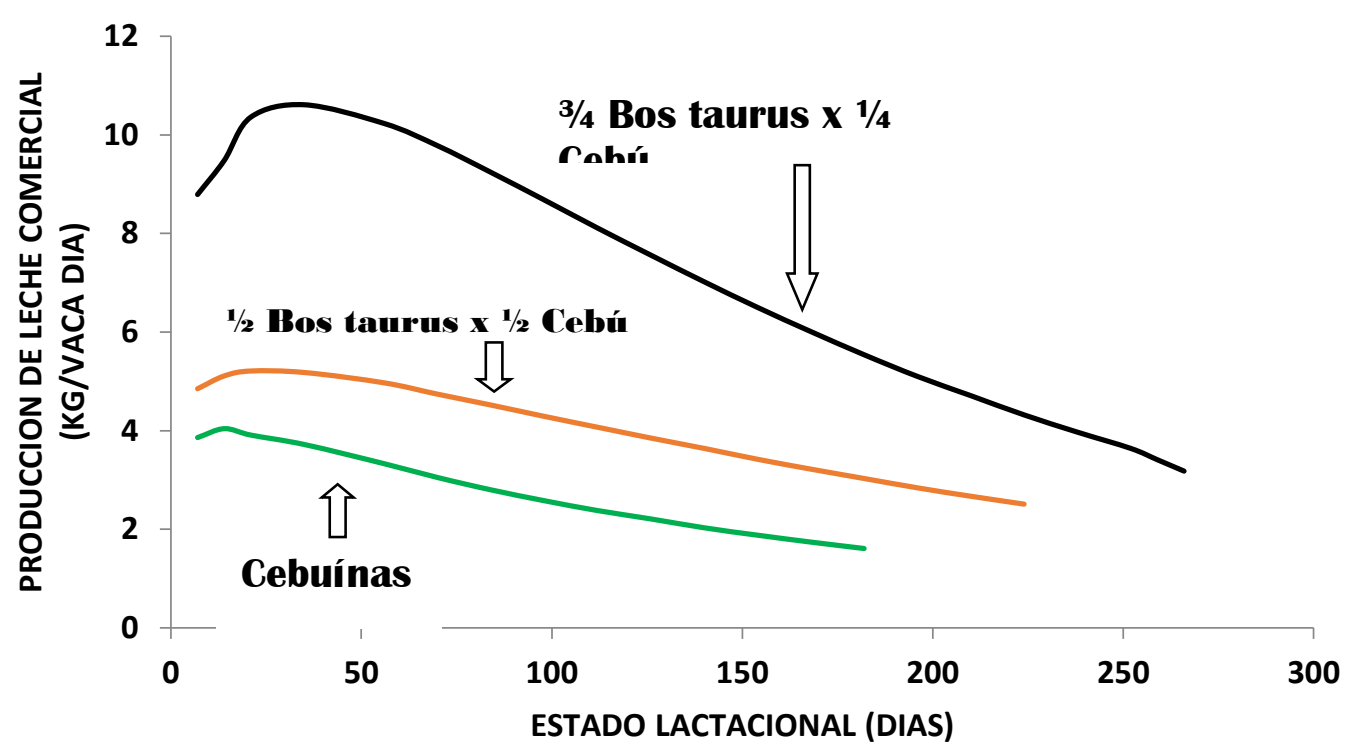

Figura 3. Trayectoria de la curva de lactación según la función logística gamma incompleta en vacas cebuínas, 1/2 Bos taurus tipo leche $x$ 1/2 Cebú y 3/4 Bos taurus $x$ 1/4 Cebú.

La forma de la curva de lactación en el grupo de las vacas multíparas $3 \frac{4}{4}$ Bos taurus x $1 / 4$ Bos indicus siguió la trayectoria con una alta aproximación al patrón lactacional en las razas lecheras Holstein, Pardo Suizo y Jersey (Visser y Wilson, 2006); aunque otros modelos sectoriales (Grossman y Koops, 1988) y polinomiales (Araúz, 2008) pueden adaptarse. La curva de lactación en las vacas cruzadas 1/2 Bos taurus lechero x 1/2 Cebú se redujo en su expresión inicial, máxima, final y en la producción total según el grupo genético.

En las vacas cebuínas multíparas se observó que la curva de lactación fue reducida en duración (177 días), producción inicial (3.42 $\pm 0.49 \mathrm{~kg}$ ), máximo de producción de $5.58 \pm 0.61 \mathrm{~kg}$ y en el cierre lactacional $2.33 \pm 0.53 \mathrm{~kg}$. Estos indicadores estuvieron asociados con el tiempo de producción con fines comerciales y el ordeño manual diario en la mañana en las fincas lecherías Grado C evaluadas en el trópico de bajura durante la época lluviosa. 


\section{CONCLUSIONES}

El modelo de producción lechera tropical con baja tecnología presentó marcadas diferencias entre los grupos genéticos, cuyo potencial lechero fue limitado por la subnutrición energética según la alimentación a base de forraje verde, la presencia el estrés calórico diurno moderado, el modelo de amamantamiento y el ordeño con ternero al pie; los cuales de manera conjunta disminuyeron la habilidad lechera fenotípica, comercial y productiva de las vacas y fincas.

Los puntos críticos y la trayectoria de la lactación en las vacas cebuínas y cruzadas fue heterogénea; pero a su vez, evidencio la importancia del aporte proporcional de la genética tipo leche con las razas Holstein y Pardo Suizo en los cruces lecheros comerciales para el medio tropical, aun bajo condiciones de tecnología limitada y en presencia de otros factores ambientales negativos como la alimentación y la presión calórica ambiental propias del medio tropical húmedo. El aumento del aporte genético del Bos taurus tipo leche en los cruzamientos incrementó los indicadores lactacionales y el rendimiento lechero comercial; incrementando la producción de leche inicial, máxima, final y la duración del periodo postpartal con valor comercial y económico en el marco operativo de las fincas lecheras a pesar de los factores limitantes.

El bajo potencial energético del forraje verde, el estrés calórico tropical, el manejo del ternero y el ordeño redujeron el potencial biológico lactacional y comercial en las vacas cebuínas y cruzadas; evidenciando que el mayor aprovechamiento productivo en las fincas Grado $\mathrm{C}$ demanda ajustes en alimentación, nutrición, microambiente y manejo para favorecer la habilidad lechera e incrementar la productividad en las fincas lecheras con bajo nivel tecnológico ubicadas en las zonas bajas del trópico húmedo, aun en la época lluviosa.

\section{AGRADECIMIENTO}

En primera instancia, se les agradece a los productores de leche Grado C del Distrito de Alanje en los corregimientos de Santo Tomas, La Pita, El Tejar, Guarumal, Mostrenco, Querébalos, la Martina y Canta Gallo por haber colaborado en esta investigacion al permitir utilizar sus facilidades físicas, los animales y el personal que labora en las fincas lecheras Grado C para el levantamiento de la información de campo; a ustedes nuestro eterno agradecimiento por el apoyo brindado.

64 IPág in a Revista Investigaciones Agropecuarias 


\section{DECLARACION DE INTERESES}

Los autores declaran que no existe conflicto entre los autores, con los productores, dentro de la Universidad de Panamá u otras instancias por haber elaborado este articulo con valor científico y técnico a partir de la investigación desarrollada originalmente por el autor principal. Por tal motivo declaramos reiteradamente que no hay diferencias sobre el contenido, conformación y orientación del documento científico presentado aquí para la Revista Científica de la Facultad de Ciencias Agropecuarias de la Universidad de Panamá.

\section{REFERENCIAS BIBLIOGRÁFICAS}

Araujo Carvalho Pedro Henrique de, Ana Luiza da Costa Cruz Borges, Ricardo Reis e Silva, Helena Ferreira Lage, Paolo Antonio Dutra Vivenza, Jose' Reinaldo Mendes Ruas, Elias Jorge Facury Filho, Rodrigo Liberio Arau'jo Palhano, Lucio Carlos Gonc salves, Iran Borges, Elo1sa de Oliveira Simões Saliba, Diogo Gonzaga Jayme, Antonio Ultimo de Carvalho. Energy metabolism and partition of lactating Zebu and crossbred Zebu cows in different planes of nutrition. 2018. Citado: 15/03/2020, Disponible: PLOS ONE | https://doi.org/10.1371/journal.pone.0202088. doi: 10.1371/journal.pone.0202088; https://www.ncbi.nlm.nih.gov/pmc/articles/PMC6097685/

disponible:

Araúz, E. E. (2019). Sectores críticos en el ciclo de vida de la hembra bovina tipo leche; cuidados biotécnicos y manejo para el buen desarrollo, salud, bienestar y productividad. Citado: 24/03/2020. Disponible: www.engormix.com

Araúz, E. E. (2018). Valor térmico de las superficies para los animales en las pérdidas calóricas pasivas. En: Fisiología de la Adaptación y Producción Animal. Carrera de Zootecnia, Departamento de Zootecnia, Facultad de Ciencias Agropecuarias, Universidad de Panamá.

Araúz, E. E. (2017). Influencia del color del pelaje sobre el comportamiento térmico corporal, cinética de la sobrecarga calórica y alteración cardiorespiratoria circadiana en vacas lecheras cruzadas (6/8 Bos taurus x 2/8 Bos indicus) bajo estrés calórico en el trópico húmedo. Citado: 05/05/2018. Disponible en http://www.veterinaria.org/revistas/redvet 2017 Volumen 18 No 7, Revista electrónica de Veterinaria - ISSN 1695-7504.

Araúz, E. E. (2010). Principales registros biológicos para evaluar la capacidad funcional de la vaca lechera y su importancia para mejorar el manejo y la eficiencia en la producción lechera. Citado: 153/2020. Disponible: www.engormix.com

Araúz. E. E. (2006). El estrés calórico y sus efectos negativos sobre la fisiología, metabolismo, reproducción y eficiencia de la producción en el ganado bovino de leche. Departamento de Zootecnia, Facultad de Ciencias Agropecuarias, Universidad de Panamá.

Araúz, E. E., Araúz, E. E. y Caballero, S. (2015). Situación actual de la producción bovina de leche en Panamá. En: Generalidades y situación actual de la ganadería en Panamá. La Ganadería en América

65 | Pág i n a

$$
\text { Revista Investigaciones Agropecuarias }
$$

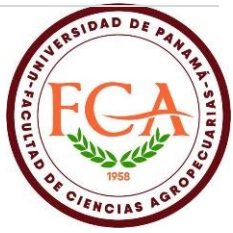


Latina y el Caribe. Editores: Rafael Núñez, Rodolfo Ramírez, Salvador Fernández, Omar Araujo, Miguel García y Tito Efraín Diaz. Pag 422 - 426. ALPA, FAO, IICA, BBA, México.

Arias, R. A., Mader, T. L. y Escoba, P. C. (2008). Factores climáticos que afectan el desempeño productivo del ganado bovino de carne y leche. Arch Med Vet 40, 7-22.

Atashi, H., Abdolmohammadi, A. R., Asaadi , A. Akhlaghi, A., Dadpasand, M. y Jafari Ahangari, Y. (2011). Using an incomplete gamma function to quantify the effect of dystocia on the lactation performance of Holstein dairy cows in Iran. Citado: 03/04/2020. https://doi.org/10.3168/jds.20114954

Cabrera, V. E. (2018). Invited review: Helping dairy farmers to improve economic performance utilizing data-driving decision support tools. Department of Dairy Science, University of WisconsinMadison, WI 53706, USA. Animal (2018), 12:1, pp 134-144 (C) The Animal Consortium 2017 doi: $10.1017 /$ S 1751731117001665.

Corella, J. (2016). Situación económica de la ganadería de leche y carne en Panamá. Conferencia en las Jornadas Agropecuarias del Banco Nacional, Bugaba, Chiriquí, República de Panamá.

Crowe M. A. y Williams E. J. (2018). Triennial lactation symposium: effects of stress on postpartum reproduction in dairy cows. Journal of Animal Science, 90,1722-7.

Curtis, E. (1981). Psychometric approach to determine heat stress in animal production. Environmental management in agriculture. USA.

Das R., L. Sailo, N. Verma, P. Bharti, J. Saikia, I. y Kumar, R. (2016). Impact of heat stress on health and performance of dairy animals: A review. Veterinary World, EISSN: 2231-0916 Citado en internet: 02 de Abril, 2020. Disponible: www.veterinaryworld.org/Vol.9/March-2016/7.

Dikmen, S. y Hansen, P. J (2009). Is the temperature - humidity in the best indicator of heat stress in lactating dairy cows in a subtropical environment? Journal of Dairy Science, 92,109 - 116.

Grossman, M. y Koops W. J. (1988). Multiphase analysis of lactation curves in dairy cattle. Journal of Dairy Science, 71, 1598.

Guerra, P. (2015). Situación actual de la ganadería de carne y las perspectivas futuras para el mejoramiento integral. En: La Ganadería en América Latina y el Caribe. Editores: Rafael Núñez, Rodolfo Ramírez, Salvador Fernández, Omar Araujo, Miguel García y Tito Efraín Diaz. Pag 4266 - 431. ALPA, FAO, IICA, BBA, México.

Gómez, J. (2002). La frecuencia del ordeño y sus ventajas. Boletín informativo, IDIAP. Estación experimental de Gualáca.

Gupta, S. K., Shinde, K. P., One, S. A., Thakur, A. y. Kumar, N. (2016). The potential impact of heat stress on production and reproduction of dairy animals: consequences and possible solutions: E-mail: sgshailesh786@gmail.com International Journal of Science, Environment ISSN 2278-3687 (O) and Technology, 5, (3), $903-911$

Hansen, P. (2009). Effects of heat stress on mammalian reproduction. Philos. Trans. Royal Society London Biologycal Science, 364(1534): 3341-3350.

Herrera, H. J. y Barreras S. A. (2000). Estadística de la Curva de lactación en la vaca de leche y el Modelo de Wood. En; Manual de Procedimientos: Análisis estadísticos de experimentos pecuarios. Montecillo, Texcoco, Estado de México, México. Citado 09/03/2020.

http://137.222.110.150/Calnet/vetrep5/image/int\%20morpholgy\%20of\%20scrotum\%20cut\%20caudally2-dog.jpg

Inalment. (2007). Cinta para la estimación del peso corporal en bovinos cebuínos, cruzados y puros por perimetría torácica. Colombia.

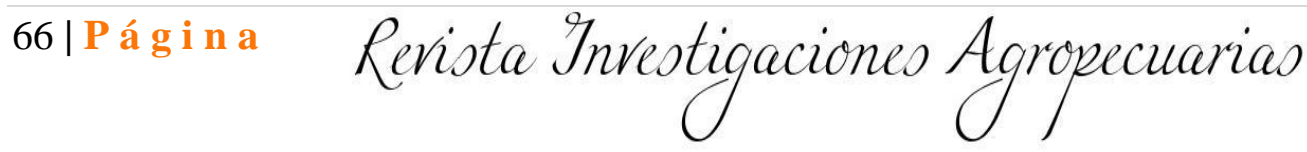


Jordan, E. R., Mader, T. L., Davis, M. S. y Brown-Brand, T. (2003). Effects of Heat Stress on Reproduction. Journal of Dairy Science, 86, E104 E114.

Knegsel, A. T. M., Van den Brand, H., Graat, E. A. M., Dijkstra, J., Jorritsma, R., Decuypere, E., Tamminga, S. y Kemp, B. (2007). Dietary Energy Source in Dairy Cows in Early Lactation: Metabolites and Metabolic Hormones. Journal of Dairy Science, 90, 1477-1485.

Knight, J. (2006). Psychrometrics calculator and evaluation of the microenvironment. Based on ASHRAE, $\begin{array}{llll}\text { Microenvironment and 26/03/2020. } & \text { Fundamentals. }\end{array}$

Disponible:https://www.kwangu.com/work/psychrometric.htm https://es.weatherspark.com/y/16739/Clima-promedio-en-Alanje-Panam\%C3\%A1-durante-todoel-a\%C3\%B1o.

Laboratorios Bayer. (2015). Boletín informativo sobre el pecutrin vitaminado como fuente de minerales. Bayer, Alemania.

Mcnab, B. (2001). Patterns of Physical exchange with the environment. En: The Physiological Ecology of Vertebrates: A View from Energetics, Publisher: Cornell University Press, NY, USA. Pag. 8 - 23.

Mader, T. L., Davis, M. S. y Brown-Brandl, B. (2006). Environmental factors influencing heat stress in feedlot cattle1,2*University of Nebraska, Concord 68728; †Koers-Turgeon Consulting Service, Inc., Salina, KS 67401; and †USDA-ARS U.S. Meat Animal Research Center, Clay Center, NE 68933.

McDowell, R. E. (1985). Crossbreeding in Tropical Areas with Emphasis on Milk, Health, and Fitness. 1985 Journal of Dairy Science, 68, 2418-2435

National Oceanic and Atmosphere Administration. 2014. Environment and Psicromteric Evaluation and heat stress. Internet, Google.

National Research Council (NRC), 1989, 2001. Nutrient Requirements of Dairy Cattle. $7^{\text {th }}$ ed., National Academy Press. Washington, DC. USA.

Nayeri, S. y Stothard, P. (2016). Tissues, Metabolic Pathways and Genes of Key Importance in Lactating Dairy Cattle. Citado: 23/03/2020. Disponible: Springer Science Reviews (2016) 4:49-77 DOI $10.1007 / \mathrm{s} 40362-016-0040-3$

Polsky, L., Marina A. y. Von Keyserlingk1, G. (2017). Invited review: Effects of heat stress on dairy cattle welfare. Animal Welfare Program, 2357 Main Mall, Faculty of Land and Food Systems, University of British Columbia, Vancouver, BC, V6T 1Z4 Canada.

Rosas, H., Gómez, J., Quintero, S. y Pimentel, N. (1985). Composición bromatológica y valor nutricional de los pastos en Panamá. En: Composición de los alimentos en Panamá y Nutricion Animal. Editorial Universitaria, Universidad de Panamá.

Rosas, H., Gómez, J. Quintero, S. y Pimentel, N. (1994). Aporte energético de las gramíneas y valor nutricional de los pastos en Panamá. En: Nutrición Animal y Composición de los alimentos en Panamá. Editorial Universitaria, Universidad de Panamá.

SAS, (1999, 2004). The analysis of variance and covariance. General Lineal Models (GLM), Statistical Analyses System, NC, USA.

Slimen, I., Belhadj, T., Najar A., Ghram y Abdrrabba, M. (2015). Heat stress effects on livestock: molecular, cellular and metabolic aspects, a review. Journal of Animal Physiology and Animal Nutrition.

Slimen, B., Najar, T., Ghram A y Abdrrabba, M. (2016). Heat stress effects on livestock: molecular, cellular and metabolic aspects. Journal of Animal Physiology and Animal Nutrition,100, 401-412.

Statistical Analysis System (SAS). (2004). General Lineal Models and the Analysis of Variance Covariance. North Caroline State University, Raleigh, NC, USA.

St-Pierre, N. R., Cobanov, B. y Schnitkey, G. (2003). Economic Losses from Heat Stress by US Livestock Industries1 *Department of Animal Sciences Journal of Dairy Science, 86, E52-E77.

67 | P á g i in a
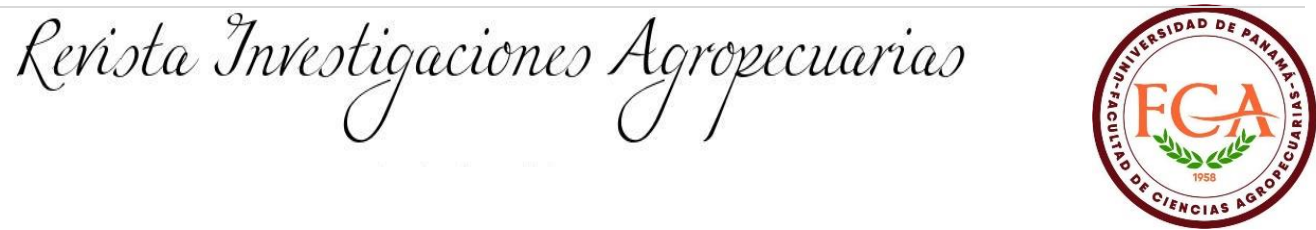
Stothard. S., Paul, A., Mandal, B., Mandal, P. Kannan, A. y. Pathak. N. N. (2004). Deriving Nutrient Requirements of Lactating Indian Cattle under Tropical Condition Using Performance and Intake Data Emanated from Feeding Trials Conducted in Different Research Institutes. Citado: 10/02/2020. Disponible: Asian-Aust. Journal of Animal Science, 2004 (6), 769-776)

Visser, R. y Wilson, R. (2006). Potencial de la producción lechera según los grupos raciales tipo leche. Horizons, CRI. Revista distribuida por Cooleche, Concepción, Bugaba, Provincia de Chiriquí.

Walker G. P., Dunshea, C. F. R. y Doyle, A. (2004). Effects of nutrition and management on the production and composition of milk fat and protein: a review. CSIRO PUBLISHING www.publish.csiro.au/journals/ajar Australian Journal of Agricultural Research, 2004, 55, 10091028. Australian Journal of Agricultural Research, 55, 1009-1028

West, J. W. (2003). Effects of Heat Stress on Production in Dairy Cattle. Journal of Dairy Science, 86:21312144.

Wood P. D. P. (1967). Algebraic models of the lactation curve in cattle. Nature, 216, 164-165. 